\title{
A Survey for Contagious Caprine Pleuropneumonia in Agago and Otuke Districts in Northern Uganda
}

\author{
Stella A. Atim1, Chrisostom Ayebazibwe1, Frank N. Mwiine², Joseph Erume², \\ Robert Tweyongyere ${ }^{2 *}$ \\ ${ }^{1}$ Ministry of Agriculture, Animal Industry and Fisheries, Entebbe, Uganda \\ ${ }^{2}$ College of Veterinary Medicine, Animal Resources and Biosecurity, Makerere University, Kampala, Uganda \\ Email: ${ }^{*}$ tmrobert966@gmail.com
}

Received 7 November 2015; accepted 11 January 2016; published 14 January 2016

Copyright (C) 2016 by authors and Scientific Research Publishing Inc.

This work is licensed under the Creative Commons Attribution International License (CC BY). http://creativecommons.org/licenses/by/4.0/

\section{Open Access}

\section{Abstract}

Background: Contagious Caprine Pleuropneumonia (CCPP) is a devastating disease of goats caused by Mycoplasma capricolum subsp. Capripneumoniae (Mccp). The disease was first confirmed in Uganda in 1995 in Karamoja region. Contagious Caprine Pleuropneumonia negatively impacts on goats' productivity but its extent and magnitude among the local communities in Uganda remain unknown. A cross sectional study was conducted in the districts of Agago and Otuke neighboring Karamoja in Northern Uganda during the months of July and August 2011 to explore for the status of the disease. Methods: Five hundred and four serum samples from goats were obtained from randomly selected unvaccinated herds and 100 goats from vaccinated herds. Serum samples were examined for antibodies against Mycoplasma capricolum subsp. Capripneumoniae (Mccp) by ELISA method. A total of 162 semi-structured questionnaires were administered to selected farmers to obtain information on their understanding of the disease and the risk factors they associated with CCPP. Eight focus group discussions were also conducted with selected farmer groups to obtain detailed qualitative information on CCPP. Results: Among the unvaccinated goats, seroprevalence of CCPP was $32(17.7 \%)$ and $52(23.3 \%)$ for Agago and Otuke districts respectively. Levels of antibodies against Mccp were higher among vaccinated goats than unvaccinated ones (mean optical densities (ODs) of 0.905 and $0.776, p=0.08$ ). Majority of the farmers $121(74.7 \%)$ had knowledge on CCPP and recognized that CCPP was among the major challenges to goat production in Uganda. Conclusions: This study demonstrated that CCPP was prevalent in Agago and Otuke districts, which are outside but close to Karamoja region where the disease was previously confirmed. There is a need for wider and detailed studies to investigate further CCPP in other districts of Uganda for effective preventive and control of CCPP in Uganda and the neighboring countries.

\footnotetext{
*Corresponding author.
}

How to cite this paper: Atim, S.A., Ayebazibwe, C., Mwiine, F.N., Erume, J. and Tweyongyere, R. (2016) A Survey for Contagious Caprine Pleuropneumonia in Agago and Otuke Districts in Northern Uganda. Open Journal of Veterinary Medicine, 6, 9-14. http://dx.doi.org/10.4236/ojvm.2016.61002 
Keywords

Contagious Caprine Pleuropneumonia, CCPP Seroprevalence, Uganda

\section{Introduction}

Contagious caprine pleuropneumonia (CСPP) is a highly infectious and devastating respiratory disease of goats caused by Mycoplasma capricolum subspecies capripneumoniae (Мсср). Clinically the diseased is characterized by coughing, respiratory distress and very high morbidity and mortality rates [1]. The disease is included in the list of notifiable diseases of World Organization for Animal Health as it threatens a significant number of goat populations throughout the world causing significant socioeconomic impact mainly in Africa and Asia.

Infection of susceptible goats is mainly through inhalation of contaminated aerosols from infected goats. Due to the high sensitivity of Mycoplasma to the external environment, close contact between infected and susceptible animals is essential for effective transmission of Mccp to take place [2], and overcrowding and confinement have been known to favors close contact and circulation of the Mycoplasma. Stress factors such as malnutrition and movement over long distances have been documented to enhance spread and morbidity of the disease [3] [4]. In Africa where extensive and traditional husbandry is practiced, pathogens have been reported to spread when animals meet at watering points and communal grazing areas.

In Africa where extensive and traditional husbandry is practiced, pathogens have been reported to spread when animals meet at watering points and grazing areas. Latent carriers such as goats or sheep that recovered from the infection without becoming bacteriological sterile were reported responsible for the perpetuation of the disease in herds [1] [5].

Clinically, the disease is characterized by high fever, respiratory distress and high mortality between $60 \%$ and $100 \%$ in absence of antibiotic intervention [1] [6] [7]. The pathological lesions are characteristic presenting interstitial fribinous pleuropneumonia, interlobular oedema and hepatization of the lung. This involvement is often unilateral, and the affected lungs are known to be port-wine coloured with possible total hepatization [1] [2]. There is involvement of the pleura with the pleural cavity containing straw-coloured exudate with fibrin flocculations [6] [8]. Contagious Caprine Pleuropneumonia (CCPP) is a disease of major economic importance in Asia and Africa, causing major constraint to goat production because of high mortalities [9] but the extent of the disease in Uganda is not well documented. In Uganda CCPP was previously confirmed in the Karamoja region [10], in the North-East of Uganda inhabited by nomadic pastoralists.

Here, we present the findings of a study carried out in two districts Agago and Otuke in Northern Uganda about CCPP and its predisposing factors in goat populations as well as the farmer's attitude about the disease. These districts are outside but close to Karamoja sub region where CCPP had been previously confirmed [10] and yet no documentation of CCPP status in the surrounding areas has been done. This preliminary finding would inform the current situation and provide information about the appropriate preventive and control measures to be instituted against CCPP in the country.

\section{Materials and Methods}

A cross sectional study was conducted in Agago and Otuke districts adjacent to Karamoja region in Northern Uganda to determine farmers' perception of CCPP and to determine the sero-prevalence of CCPP in these areas. To obtain information on CCPP and other goat diseases in the areas, a structured questionnaire was administered to randomly selected goat farmers and focused group discussions conducted with eight farmers groups using participatory epidemiological methods.

\section{Indirect ELISA for CCPP}

To determine the sero-prevalence of CCPP, antibodies against CCPP were measured for serum samples from 504 goats using an in-house indirect ELISA. The ELISA for detection of antibodies against Mccp was developed, optimized and performed in the Central Diagnostic Laboratory at the College of Veterinary Medicine, Animal resources and Biosecurity, Makerere University Kampala, Uganda. 
Briefly: ELISA plate, (NUNC Immunoplate 439,454, Thermo Fisher Scientific, Roskilde, Denmark) were coated with $100 \mu \mathrm{L}$ per well of inactivated Mccp antigen (Caprivax, Kenya Veterinary Vaccines Production Institute, Nairobi, Kenya) at 1:100 dilution and incubated overnight in at $40 \mathrm{c}$. The plates were washed, $150 \mu \mathrm{L}$ per well of blocking buffer (2\% bovine sarum albumin (BSA) in Phosphate buffer saline (PBS)) added and incubated at room temperature for one hour. Test serum samples were diluted at 1:100 in assay diluent (1\% BSA + $0.1 \%$ Tween 20 in PBS) and added $100 \mu \mathrm{L}$ per well. Known positive sera (strong and weak positive) and negative sera were added on each plate. The plates were incubated at room temperature for one hour. Conjugate, protein G HRPO at 1:5000 dilutions was added $100 \mu \mathrm{L}$ per well and incubated at room temperature for one hour. ABTS substrate was added $100 \mu \mathrm{L}$ per well and incubated at room temperature for 10 minutes. The reaction was stopped after 30 minutes incubation by addition of $1 \%$ Sodium dodecylsulphate (VWR BDH) and the optical densities (ODs) read at $450 \mathrm{~nm}$.

An arbitrary cutoff based on the negative control included on each plate was set at 3 standard deviations above the mean OD value of the negative control. The mean OD for the negative sample was 0.1133 and $\mathrm{SD}=$ 0.036; hence the cutoff was 0.241 . An arbitrary categorization of the results was made as negative (OD $<0.25)$, trace (OD $0.25<0.5$ ), weak positive (OD $0.5<1$ ) or strong positive OD $>1$ ). To compute the sero-prevalence, weak and strong positives were considered as positive while those with trace were considered with the negative.

The questionnaire data was entered using EpiDatasoft ware and later imported into stata (Version 9.1) for analysis. The ELISA reading data was automatically recorded into Microsoft excels 2007 and later imported into stata for analysis. The qualitative data from the questionnaire and focus group discussions were analysed thematically and a code sheet to show sequence in responses were developed accordingly. Descriptive statistics including percentages, chi-square test $p$-values are presented.

\section{Results}

\subsection{Sero-Prevalence of CCPP in the Districts of Agago and Otuke}

Serum samples were obtained from 404 goats from herds with no history of CCPP vaccination and 100 goats vaccinated for CCPP by MAAIF in the last four months before sample collection. Of the unvaccinated goats sampled, 181 were from Agago district and 223 from Otuke district.

Antibody titers (in terms of ODs) were compared between vaccinated and unvaccinated goats. Of note was that although the antibodies among the vaccinated goats generally tended to be higher than unvaccinated goats this difference was not statistically significant, $p=0.08$ (Figure 1). The sero-prevalence of CCPP was $20.8 \%$ and was not significantly different between Agago (17.7\%) and Otuke (23.3\%) districts (Chi square $p=0.165$ ).

\subsection{Farmers' Perceptions on Diseases of Goats and Knowledge of CCPP in Agago and Otuke}

Questionnaires were administered to 162 individual goat farmers and focused group discussions were conducted with eight farmers' groups. Common goat diseases in the study area in order of importance as listed by the

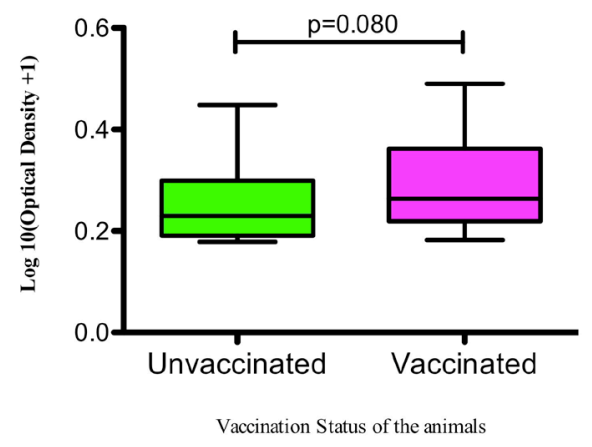

Figure 1. Levels of antibodies to Mccp antigen compared between the unvaccinated and vaccinated cattle. Shown on the $y$-axis are the log 10 transformed optical densities. Shown is the Wilcoxon rank-sum (Mann-Whitney) test $p$ value. 
farmers included endoparasites (39.7\%), CCPP (17.9\%), mange (16.2\%), orf (13.5\%), tick infestations (12.7\%) for Agago district. Similarly in Otuke districts endoparasites (34.2\%), CCPP (25.5\%), tick infestations (20.5\%), mange (13.7) and orf (6.2\%) were highlighted as the major health challenges in goat rearing. Similar patterns were equally obtained in the proportionate ranking of common goat diseases in the FGDs with the farmers' groups.

Of the 162 farmers who responded to the questionnaire, 121 (74.7\%) were aware of CCPP and gave the local luo language name of CCPP as "two-oboodyel" or "loukoidyel" (Agago) or "two-iwukudyel" (Otuke). Of the 121 farmers who were aware of CCPP, 86 (82.6\%) reported to have had the disease in their herds. They emphasized that the disease was recognized from clinical signs, which included cough/difficult breathing, fever, loss of appetite, nasal discharge, difficult movement and loss of body condition that often culminated into death of the animal. Further, the farmer groups were also able to point out seasonal pattern of CCPP during the participatory focused group discussions. It was apparent that CCPP is understood to be more prevalent in the months of July to August of the annual calendar.

Of the respondent farmers, 86 (82.6\%) who reported to have encountered CCPP in their herds, 49 (57\%) sought for professional veterinary care for the affected goats (Table 1). Surprisingly, 25 (29.1\%) of the respondents reported that they never took any particular action in the care of their sick goats while $12(14.0 \%)$ reported to have only applied local herbs as a form of treatment.

It was also noted during the discussion that farmers pointed out herd sizes, farming practices and source of breeding stock as risk factors for occurrence of CCPP. Of the 86 farmers who reported to have encountered CCPP, 74 (86.1\%) attributed the disease to rearing small herd sizes managed by communal grazing. Most of the farmers $(65(75.6 \%))$ also pointed out that practicing communal grazing with seldom tethering is likely to increase CCPP infections and spread When farmers were asked on the risk of CCPP infections from breeding stocks, 55 (64\%) of the farmers were aware of the risk of introducing the infection and preferred to acquire breeding stocks from far off districts they considered free of the disease.

\section{Discussions}

The main aim of this survey was to explore the occurrence of CСPP beyond the boarders of Karamoja region where the disease had been previously confirmed [10]. This study confirmed that indeed CCPP negatively impact on goat health and production in Agago and Otuke but its extent and magnitude among local communities remains to be fully elucidated. The ranking of CCPP by farmers in Agago and Otuke as second among the health challenges of their goats corroborates with Southern Ethiopia participatory investigation of CCPP where farmers ranked the disease major constraint in goat production [11]. The clinical signs and postmortem lesions identified by farmers in Agago and Otuke were closely related to earlier documented clinical signs of the disease [2] [12].

The seasonal pattern of CCPP identified by farmers during onset of rains agreed with previous reports from Southern Ethiopia [11]. This also affirmed previous reports that seasonal calendars were important tool in designing disease control programs [13] [14] and this could be equally targeted as best time for control of CCPP in Agago and Otuke districts.

The farmers' attitude towards treatment of CCPP in Agago and Otuke was largely related to use of modern veterinary drugs than use of local herbs even though a few used both. This observation is of interest and contrary to previous reports that purely pastoral communities depend highly on local materials mainly plants to manage livestock health problems as first line of treatment [15] [16]. However, of note was that up to $29.1 \%$ of the farmers did not take any action against CCPP infections in their herds and this could probably signify the low value some farmers still attached to small ruminant.

Table 1. Farmers' approach to management of cases of Contageous Caprine Pleuropneumonia.

\begin{tabular}{ccccc}
\hline District & $\begin{array}{c}\text { Sought veterinary service } \\
\text { and applied veterinary drugs }\end{array}$ & Use of local herbs & $\begin{array}{c}\text { Sought veterinary help and } \\
\text { also applied local herbs }\end{array}$ & No action taken \\
\hline Agago $(\mathrm{n}=45)$ & $19(42.2 \%)$ & $8(17.8 \%)$ & $2(4.4 \%)$ & $16(35.6 \%)$ \\
Otuke $(\mathrm{n}=41)$ & $22(53.7 \%)$ & $4(9.8 \%)$ & $6(14.6 \%)$ & $9(22.0 \%)$ \\
Overall & $41(47.7 \%)$ & $12(13.9 \%)$ & $8(9.3 \%)$ & $25(29.1 \%)$ \\
\hline
\end{tabular}


All the goat husbandry practices in Agago and Otuke were still traditional irrespective of herd sizes reared by farmers, source of breeding stock and farming practices. Such traditional husbandry practices were previously reported to favor spread of CCPP when animals meet at watering points and grazing areas because of increased contact rates between infected goats and naïve ones essential for effective transmission of Mccp [2]-[4].

The present study estimated the overall sero-prevelance of CCPP at 20.8\%. This is close to what has been reported (15\% - 18\%) in southern Ethiopia [4] [11]. Our finding was at variance with reports of studies done in Ethiopia [17] [18] and in Beetal goats in Pakistan Hussain, 2012 \#51 as well as in East Turkey [19] who documented higher sero-prevalence between $31 \%$ and $38 \%$. The sero-prevalence of the disease was not significantly different between the two districts, Agago (17.7\%) and Otuke (23.3\%). This is not surprising since Agago and Otuke lie within the same agro ecological zone, a similar explanation earlier reported in Ethiopia [18].

Important to note was that comparison of antibody levels (ODs) among the vaccinated goats were slightly higher than unvaccinated but the difference still insignificant $(p=0.08)$. We expected a significant difference in the antibody levels between two. This may suggest that vaccination may not be provoking sufficient seroconversion thus putting the efficacy of this vaccine into question. Never the less, this findings were in disagreement with high efficacy reported with inactivated Mycoplasma strain F38 saponin vaccine in natural infection with CCPP [20]-[22] and experimentally lyophilized killed F38 vaccine that conferred $100 \%$ protection against mortality and 95\% clinical disease of Mycoplasma species strains F38 [23]. Seroprevalence done in Tanzania [24] and in Ethiopia [4] [18] have also shown no sex difference in CCPP epidemiology.

\section{Conclusions}

The preliminary questionnaire survey, focus group discussions and the serological findings strongly demonstrated that CCPP is prevalent in Agago and Otukedistricts, which are outside but close to Karamoja region where the disease was previously confirmed. This study also showed most farmers to be aware of CCPP and the use of participatory disease investigations approaches which could be of relevancy in designing and timing CCPP control programs in these districts.

The study covered only Agago and Otuke; therefore, more detailed and bigger studies should be undertaken to investigate further CCPP in rest of the districts in Uganda to pave way for effective preventive and control measures against CCPP in the country. Secondly, there is a need to develop a diagnostic test which will be easy to use and readily available in Uganda.

\section{Acknowledgements}

The authors would like to appreciate the team at Ministry of Agriculture, Animal Industry and Fisheries NADDEC for the great work both in the field during the sample collection and in the laboratory analysis of the samples. Dr. Thomson Anyuru DVO Otuke and Mr. Paul Oneka DVO Agago. This work was facilitated by institutional, financial and material support from MAAIF, Makerere University, and AFRUS-IDM (HED/ CIMTRADZ) project.

\section{References}

[1] Thiaucourt, F. and Bolske, G. (1996) Contagious Caprine Pleuropneumonia and Other Pulmonary Mycoplasmoses of Sheep and Goats. Revue Scientifique et Technique, 15, 1397-1414.

[2] Thiaucourt, F., et al. (1996) Diagnosis and Control of Contagious Caprine Pleuropneumonia. Revue Scientifique et Technique, 15, 1415-1429.

[3] Lefevre, P.C., et al. (1987) Mycoplasma Species F 38 Isolated in Chad. Veterinary Record, 121, 575-576.

[4] Mekuria, S. and Asmare, K. (2010) Cross-Sectional Study on Contagious Caprine Pleuro Pneumonia in Selected Districts of Sedentary and Pastoral Production Systems in Southern Ethiopia. Tropical Animal Health and Production, 42, 65-72. http://dx.doi.org/10.1007/s11250-009-9386-8

[5] Wesonga, H., et al. (1993) Relationship between Clinical Signs and Early Lesions of Contagious Caprine Pleuropneumonia Caused by Mycoplasma Strains F38. Small Ruminant Research, 10, 45-54. http://dx.doi.org/10.1016/0921-4488(93)90106-R

[6] Kaliner, G. and MacOwan, K.J. (1976) The Pathology of Experimental and Natural Contagious Carine Pleuropneumonia in Kenya. Zentralblatt fur Veterinarmedizin. Reihe B, 23, 652-661. http://dx.doi.org/10.1111/j.1439-0450.1976.tb00703.x 
[7] Jones, G.E. and Wood, A.R. (1988) Microbiological and Serological Studies on Caprine Pneumonias in Oman. Research in Veterinary Science, 44, 125-131.

[8] Wesonga, H.O., et al. (1998) Late Lesions of Experimental Contagious Caprine Pleuropneumonia Caused by Mycoplasma capricolum ssp. Capripneumoniae. Zentralblatt fur Veterinarmedizin. Reihe B, 45, 105-114.

[9] Rurangirwa, F.R., Masiga, W.N. and Muthomi, E.K. (1984) Immunisation of Goats against Contagious Caprine Pleuropneumonia Using Sonicated Antigens of F-38 Strain of Mycoplasma. Research in Veterinary Science, 36, 174-176.

[10] Bolske, G., et al. (1995) Contagious Caprine Pleuropneumonia in Uganda and Isolation of Mycoplasma capricolum Subspecies Capripneumoniae from Goats and Sheep. Veterinary Record, 137, 594.

[11] Mekuria, S., Zerihun, A., Gebre-Egziabher, B. and Tibbo, M. (2008) Participatory Investigation of Contagious Caprine Pleuropneumonia (CCPP) in Goats in the Hammer and Benna-Tsemay Districts of Southern Ethiopia. Tropical Animal Health and Production, 40, 571-582. http://dx.doi.org/10.1007/s11250-008-9136-3

[12] Wesonga, H.O., Bölske, G., Thiaucourt, F., Wanjohi, C. and Lindberg, R. (2004) Experimental Contagious Caprine Pleuropneumonia: A Long Term Study on the Course of Infection and Pathology in a Flock of Goats Infected with Mycoplasma capricolum subsp. capripneumoniae. Acta Veterinaria Scandinavica, 45, 167-179. http://dx.doi.org/10.1186/1751-0147-45-167

[13] Catley, A., Irungu, P., Simiyu, K., et al. (2002) Participatory Investigations of Bovine Trypanosomiasis in Tana River District, Kenya. Medical and Veterinary Entomology, 16, 55-66. http://dx.doi.org/10.1046/j.0269-283x.2002.00346.x

[14] Catley, A., Alders, R.G. and Wood, J.L. (2012) Participatory Epidemiology: Approaches, Methods, Experiences. The Veterinary Journal, 191, 151-160. http://dx.doi.org/10.1016/j.tvjl.2011.03.010

[15] Githiori, J.B., Hoglund, J. and Waller, P.J. (2005) Ethnoveterinary Plant Preparations as Livestock Dewormers: Practices, Popular Beliefs, Pitfalls and Prospects for the Future. Animal Health Research Reviews, 6, 91-103. http://dx.doi.org/10.1079/AHR2005099

[16] Giday, M. and Teklehaymanot, T. (2013) Ethnobotanical Study of Plants Used in Management of Livestock Health Problems by Afar People of Ada'ar District, Afar Regional State, Ethiopia. Journal of Ethnobiology and Ethnomedicine, 9, 8. http://dx.doi.org/10.1186/1746-4269-9-8

[17] Eshetu, L., Yigezu, L. and Asfaw, Y. (2007) A Study on Contagious Caprine Pleuropneumonia (CCPP) in Goats at an Export Oriented Abattoir, Debrezeit, Ethiopia. Tropical Animal Health and Production, 39, 427-432. http://dx.doi.org/10.1007/s11250-007-9041-1

[18] Hadush, B., Eshetu, L., Mengistu, W. and Hailesilassie, M. (2009) Seroprevalence of Contagious Caprine Pleuropneumonia in Kefta Humera, Alamata (Tigray) and Aba-'ala (Afar), Northern Ethiopia. Tropical Animal Health and Production, 41, 803-806. http://dx.doi.org/10.1007/s11250-008-9255-X

[19] Cetinkaya, B., Kalin, R., Karahan, M., et al. (2009) Detection of Contagious Caprine Pleuropneumonia in East Turkey. Revue Scientifique et Technique, 28, 1037-1044.

[20] Rurangirwa, F.R., McGuire, T., Kibor, A. and Chema, S. (1987) An Inactivated Vaccine for Contagious Caprine Pleuropneumonia. Veterinary Record, 121, 397-400. http://dx.doi.org/10.1136/vr.121.17.397

[21] Litamoi, J.K., Lijodi, F.K. and Nandokha, E. (1989) Contagious Caprine Pleuropneumonia: Some Observations in a Field Vaccination Trial Using Inactivated Mycoplasma Strain F38. Tropical Animal Health and Production, 21, 146150. http://dx.doi.org/10.1007/BF02236196

[22] King, G.J., Kagumba, M. and Kariuki, D.P. (1992) Trial of the Efficacy and Immunological Response to an Inactivated Mycoplasma F38 Vaccine. Veterinary Record, 131, 461-464. http://dx.doi.org/10.1136/vr.131.20.461

[23] Rurangirwa, F.R., McGuire, T.C., Mbai, L., et al. (1991) Preliminary Field Test of Lyophilised Contagious Caprine Pleuropneumonia Vaccine. Research in Veterinary Science, 50, 240-241. http://dx.doi.org/10.1016/0034-5288(91)90114-4

[24] Kusiluka, L.J., Semuguruka, W.D., Kazwala, R.R., et al. (2000) Demonstration of Mycoplasma capricolum subsp. capripneumoniae and Mycoplasma mycoides subsp. mycoides, Small Colony Type in Outbreaks of Caprine Pleuropneumonia in Eastern Tanzania. Acta Veterinaria Scandinavica, 41, 311-319. 